\title{
Young's Modulus and Coefficient of Linear Thermal Expansion of ZnO Conductive and Transparent Ultra-Thin Films
}

\author{
Naoki Yamamoto, Hisao Makino, and Tetsuya Yamamoto \\ Research Institute, Kochi University of Technology, Tosayamada-cho, Kochi 782-8502, Japan \\ Correspondence should be addressed to Naoki Yamamoto, yamamoto.naoki@kochi-tech.ac.jp \\ Received 29 March 2011; Accepted 17 May 2011 \\ Academic Editor: Kin Ho Lo
}

Copyright ( $) 2011$ Naoki Yamamoto et al. This is an open access article distributed under the Creative Commons Attribution License, which permits unrestricted use, distribution, and reproduction in any medium, provided the original work is properly cited.

\begin{abstract}
A new technique for measuring Young's modulus of an ultra-thin film, with a thickness in the range of about $10 \mathrm{~nm}$, was developed by combining an optical lever technique for measuring the residual stress and X-ray diffraction for measuring the strain in the film. The new technique was applied to analyze the mechanical properties of Ga-doped ZnO (GZO) films, that have become the focus of significant attention as a substitute material for indium-tin-oxide transparent electrodes. Young's modulus of the as-deposited GZO films decreased with thickness; the values for $30 \mathrm{~nm}$ and $500 \mathrm{~nm}$ thick films were $205 \mathrm{GPa}$ and $117 \mathrm{GPa}$, respectively. The coefficient of linear thermal expansion of the GZO films was measured using the new technique in combination with in-situ residual stress measurement during heat-cycle testing. GZO films with 30-100 nm thickness had a coefficient of linear thermal expansion in the range of $4.3 \times 10^{-6}-5.6 \times 10^{-6}{ }^{\circ} \mathrm{C}^{-1}$.
\end{abstract}

\section{Introduction}

Optically transparent and conductive $\mathrm{ZnO}$ films, such as $\mathrm{Ga}-$ doped Zinc oxide (GZO) or Al-doped ZnO films, are attracting attention as alternative electrode materials to indium tin oxide (ITO), with good optical transparency and electrical conductive properties for liquid crystal displays (LCDs), flexible displays, touch-screens, and solar cells. Motivations for the development of $\mathrm{ZnO}$ transparent electrodes include (a) the scarcity of indium natural resources and (b) the toxicity of indium. Indium is a rare element and ranks only 61st in abundance in the Earth's crust [1]. Also, in terms of toxicity, recently, indium was suspected to be a cause of pulmonary diseases [2].

$\mathrm{ZnO}$ electrodes need to endure against mechanical forces caused by the thermal processing steps during the manufacture of electronic devices such as flat panel displays, flexible displays, touch-screens, and LEDs, and by the expansion or shrinkage of parts caused by thermally severe circumstances (during summer and winter, e.g.) in the case of solar cells. These external forces could enhance the frequency of peeling or cracking in the elements composing the systems, such as transparent electrodes, during the fabrication or operation of the devices. It is desired that the films used to form such electrodes have mechanically soft properties, because soft materials can absorb external forces and avoid peeling and cracking of the films. To obtain such a film, various mechanical characteristics of the thin film, such as elasticity, plasticity, viscosity, brittleness, yield strength, and adhesiveness, should be evaluated and be analyzed.

In these characteristics, Young's modulus is one of the most important mechanical properties of a film. It is an intrinsic property of the film (material) that relates to the atomic bonding and the crystalline structure.

In this work, a new technique was developed for measuring the mechanical characteristics of an ultra-thin film of a transparent electrode in LCD. Young's moduli and the coefficients of linear thermal expansion of GZO thin films were analyzed using the new technique.

\section{Experimental}

GZO films were prepared using two deposition methods: (1) conventional magnetron sputtering with dc power (dc MS) or with combined dc power/radiofrequency (rf) sputtering $(\mathrm{rf}+\mathrm{dc} \mathrm{MS})$, and (2) reactive plasma deposition (RPD) [3]. 
The concentration of gallium in the deposition source was set to be $3.0-6.0 \mathrm{wt} \% \mathrm{Ga}_{2} \mathrm{O}_{3}$ in order to minimize the GZO film resistivity. The conditions used for preparation of the GZO films are listed in Table 1.

The residual stress in the films was measured by the conventional optical lever method (model: F2300, Flexus Co.), which involved scanning a $633 \mathrm{~nm}$ wavelength laser beam of an HeNe laser (CW mode, laser power: $4 \mathrm{~mW}$ ). A sample was put on the 3 small surface-projections arranged at the positions of the equilateral triangle on the heater plate. The residual stress in the film was measured during heating from 25 to $500^{\circ} \mathrm{C}$ and then cooling to $25^{\circ} \mathrm{C}$. The substrate was deformed by the residual stress in the film. The radius of curvature of the substrate was measured by detecting the reflected laser beam. The residual stress in the film was then obtained by measuring the change in the curvature radius of the substrate before and after forming the film on it, and by using the coefficient of linear thermal expansion and Young's modulus of the substrate.

The crystal structures of the GZO films were analyzed using an X-ray diffractometer (XRD, model: ATX-G Rigaku Co.), a transmission electron microscope (TEM model: $\mathrm{H}$ 9000UHR Hitachi High-technologies Co.) and a scanning electron microscope (SEM, model: S-4300 Hitachi Hightechnologies Co.).

\section{Results and Discussion}

\subsection{Residual Stress in GZO Thin Films}

3.1.1. Residual Stress in GZO Films as a Function of Thickness. The crystalline structure of the GZO films was observed using a TEM. Figure 1 shows a cross-sectional TEM image of a $100 \mathrm{~nm}$ thick GZO film formed at $180^{\circ} \mathrm{C}$ by the RPD system. The GZO film has a columnar polycrystalline structure, as shown in the low magnification TEM micrograph in Figure 1(a). The alternating black and white striped layers in the middle magnification micrograph in Figure 1(b) correspond to $\mathrm{Zn}$ and $\mathrm{O}$ atomic layers in a Wurtzite crystalline structure forming the GZO material. The irregular parts in the image with stacked $\mathrm{Zn}$ and $\mathrm{O}$ atomic layers can be recognized in the vicinity of the surface of the substrate (region (1) in the lowest magnification micrograph (a)) as shown in Figure 1(b). This irregularity disappeared from a distance of approximately $50 \mathrm{~nm}$ from the surface of the substrate. The GZO film had an excellent crystalline structure, similar to a single crystal, as shown in the cross-sectional lattice image in region (2) in Figure 1(a) with distance from the surface of the substrate as shown in Figure 1(c). The boundary position in the cross-sectional lattice image cannot be identified.

Generally, stress increases remarkably at a dispersive location such as a surface or a discontinuity in a material. We forecasted that the residual stress in a GZO film with ca. $50 \mathrm{~nm}$ thickness would show an abnormality or irregularity on the dependence of the residual stress as a function of the film thickness. Figure 2 shows the typical residual stress dependence of the GZO film on the thickness. The residual stress of the film was measured at ca. $25^{\circ} \mathrm{C}$ in air. The films were formed on a (100) Si single crystal substrate (wafer) and a thermally oxidized silicon dioxide film at $180^{\circ} \mathrm{C}$ by the RPD system.

All the films formed in this work had compressive stress. It is known that the residual stress in a film depends on the degree of energetic particle bombardment, that is, energy striking the condensing film, during deposition in sputtering or ion-plating with plasma discharge [4-9]. RPD is a kind of ion-plating method. Energy particle bombardment, "atomic peening effect", introduces compressive stress in a film. The energy is a function of various process parameters such as working pressure, discharge voltage, gas mass, and substrate temperature.

The irregularities in the dependence of the residual compressive stress of the GZO films on the thickness appeared at approximately $60 \mathrm{~nm}$ thickness of the films formed on the Si wafer and the $\mathrm{SiO}_{2}$ layer. This thickness corresponds to a distance of ca. $50 \mathrm{~nm}$ from the surface of the substrate, indicating that a boundary between region (1) and region (2) shown in Figure 1(a) exists. This correspondence proved the close connection between the residual stress and the distribution of crystalline irregularities in the film. This indicates that residual stress measurement, one of the available macroscopic evaluation methods, can be used as a monitor of the crystal structure in the nanoscale regime in the film. Figure 3 compares the dependence of the residual stresses on thickness for the GZO films formed by dc MS, rf $+\mathrm{dc}$ MS, and RPD. It was found that the region with crystalline irregularities in the vicinity of the interface for the film formed by $\mathrm{rf}+\mathrm{dc}$ MS was distributed up to a distance of approximately $100 \mathrm{~nm}$ from the surface of the substrate. This distance was about twice longer than that for the film formed by RPD.

3.1.2. Hysteresis Behavior of Stress-Temperature Characteristics of GZO Films. The properties of the residual (compressive) stresses in GZO films formed by RPD and magnetron sputtering methods with various thicknesses, shown in Figures 4 and 5, were analyzed using heat-cycle testing. Such testing for GZO films was also discussed in our previous papers $[10,11]$. Testing between room temperature and $500^{\circ} \mathrm{C}$ was carried out twice for each sample. The heating and cooling rates were controlled to about $2.8^{\circ} \mathrm{C} / \mathrm{min}$. It is considered that these rates realized a thermally quasi-static (thermal equilibrium) situation for the samples. In-situ measurements of residual stresses in the films during the heat-cycle testing were carried out. Measurement of the residual stress took less than a few milliseconds at each point in the heat-cycle testing. Each film showed the following common behavior: (a) the compressive residual stress in the film was reduced steeply beginning from 200 to $400^{\circ} \mathrm{C}$ (the temperature differed among the films formed by dc MS, rf $+\mathrm{dc}$ MS, and RPD) when the temperature was increased in the first heating step (step (1)), (b) the residual stress decreased monotonically when the temperature was lowered from $500^{\circ} \mathrm{C}$ (step (2)), and (c) the dependence of the residual stress on the temperature in each film during the second heat-cycle (steps (3) and (4)) almost coincided with that in step (2). The linear relationship between the residual stress and temperature in steps (2)-(4) indicates that the 
TABLE 1: Specifications for the formation of GZO films by dc MS, $\mathrm{rf}+\mathrm{dc}$ MS, and RPD.

\begin{tabular}{|c|c|c|c|}
\hline & \multicolumn{2}{|c|}{ Magnetron sputtering } & \multirow{2}{*}{ RPD } \\
\hline & dc MS & $\mathrm{rf}+\mathrm{dc} \mathrm{MS}$ & \\
\hline $\mathrm{Ga}_{2} \mathrm{O}_{3}$ content in $\mathrm{ZnO}$ source (wt $\%$ ) & \multicolumn{2}{|r|}{$\mathrm{Ga}_{2} \mathrm{O}_{3}: 3.0-6.0$} & $\mathrm{Ga}_{2} \mathrm{O}_{3}: 3.0-5.0$ \\
\hline \multirow{3}{*}{ Power $(\mathrm{kW})$} & \multirow{3}{*}{$0.1-2.0$} & rf: $0.1-1.5$ & Discharge \\
\hline & & dc: $0.1-1.5$ & current: $140-150$ \\
\hline & & $\mathrm{rf} / \mathrm{dc}=0.5-2.0$ & (A) \\
\hline Operation pressure $(\mathrm{Pa})$ & $0.1-0.8$ & $0.1-0.8$ & $0.4-0.6$ \\
\hline Deposition rate $(\mathrm{nm} / \mathrm{min})$ & $4-20$ & $4-20$ & $150-170$ \\
\hline Operation temperature $\left({ }^{\circ} \mathrm{C}\right)$ & $150-350$ & $150-350$ & $150-250$ \\
\hline
\end{tabular}

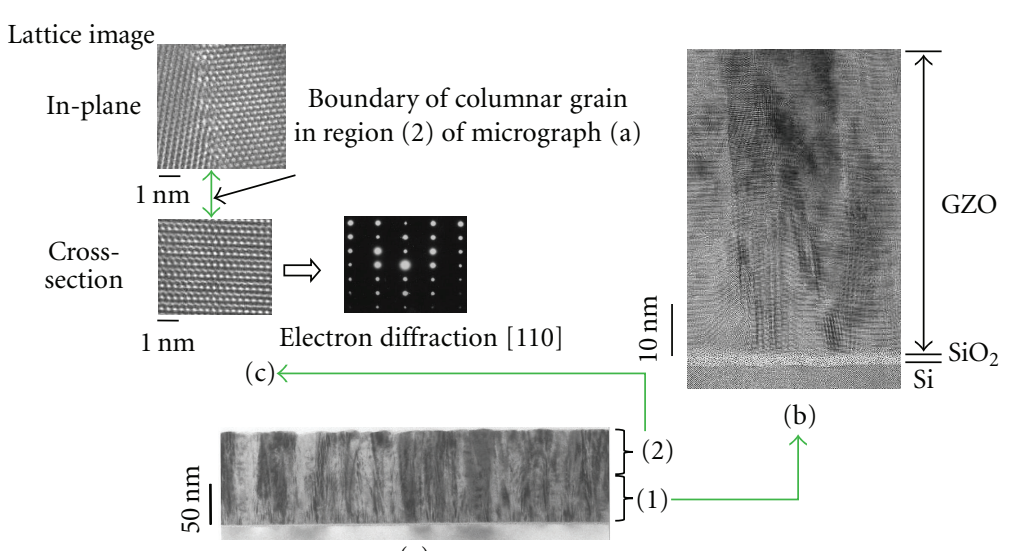

(a)

FIgure 1: (a) Cross-sectional TEM micrograph of a GZO film. (b) Close-up view of a region in the vicinity of the film-substrate interface (region (1) in (a)). The layered structure in a GZO film with a Wurtzite structure, enhanced using Photoshop Elements 3.0 software (Adobe Systems Inc.). (c) Lattice image and electron diffraction pattern observed by TEM in the vicinity of the surface of the film (region (2) in (a)).

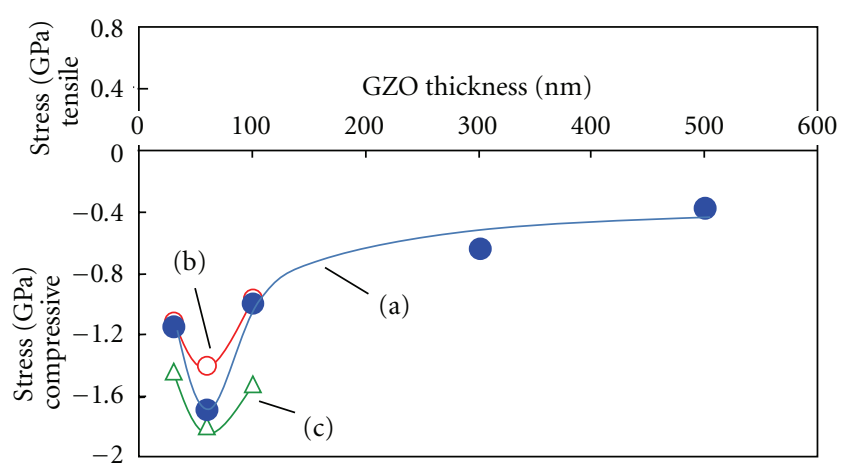

Figure 2: Dependence of residual stress in the GZO film formed by the RPD system on the film thickness before heat treatment. The symbols denote measured data. The dots (a) and open (b) circles correspond to the 1st and 2nd experimental series of GZO films respectively, and the triangles (c) show the residual stress in $\mathrm{ZnO}$ films without $\mathrm{Ga}$ (3rd series). The films in the 1st and 3rd series were formed on Si wafers with thermally oxidized silicon layer films.

major residual stress component in each film was thermal stress. This thermal stress is caused by the difference in the coefficient of linear thermal expansion between the GZO film and the Si substrate. The residual stress behavior in step (1) for the films prepared by RPD and dc MS was

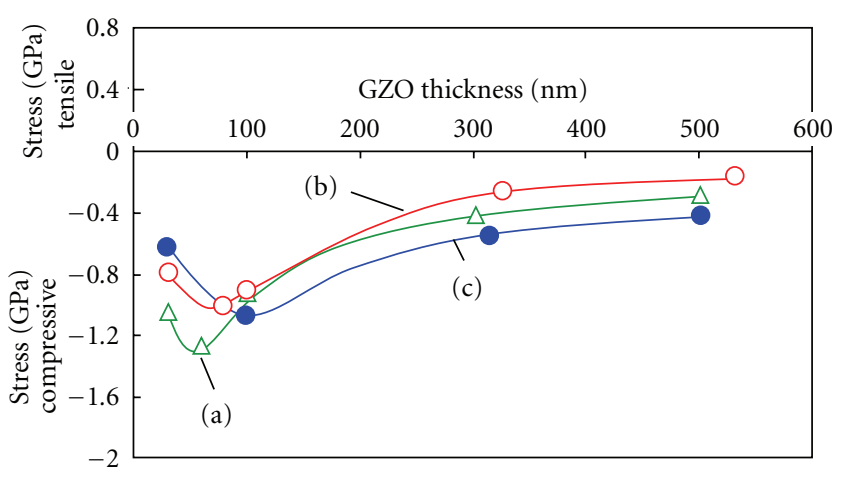

Figure 3: Comparison of the dependence of residual stress on film thickness in as-deposited GZO films prepared at $180^{\circ} \mathrm{C}$ by (a) RPD, (b) dc MS, and (c) rf + dc MS.

closer to that during steps (2)-(4) for comparatively thicker (i.e., $500 \mathrm{~nm}$ ) GZO films. Thus, the main component of the residual stress in such thick films was thermal stress (intrinsic stress), even before the heat-cycle testing for the as-deposited film. The GZO films approached the ideal crystalline structure with increasing thickness. On the other hand, the residual stress behavior in step (1) in the GZO films prepared by $\mathrm{rf}+\mathrm{dc}$ MS was obviously not close to that in steps (2)-(4) even in the $500 \mathrm{~nm}$ thick films. This 


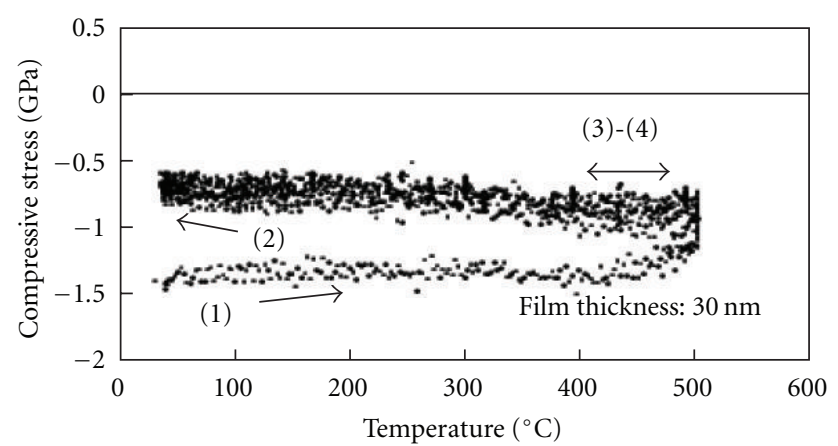

(a)

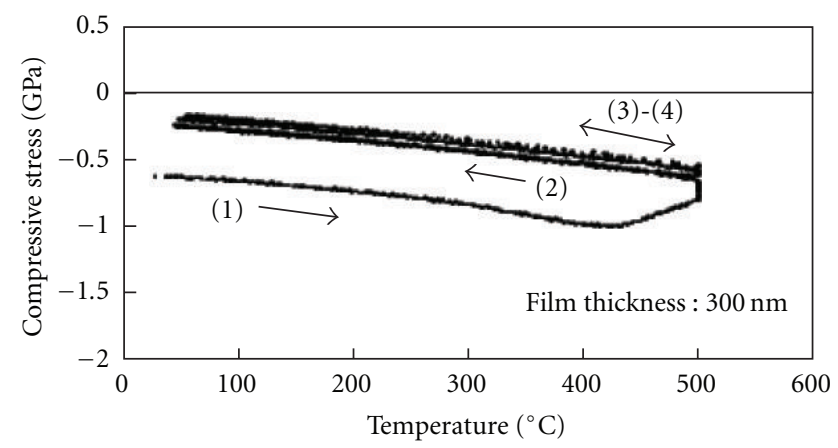

(c)

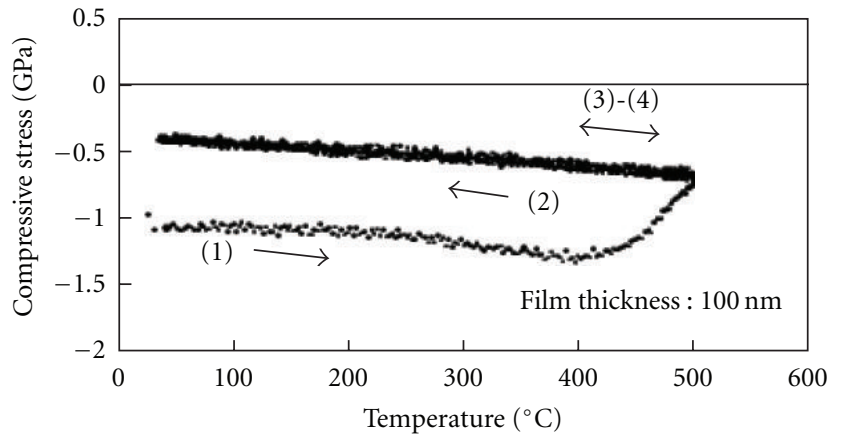

(b)

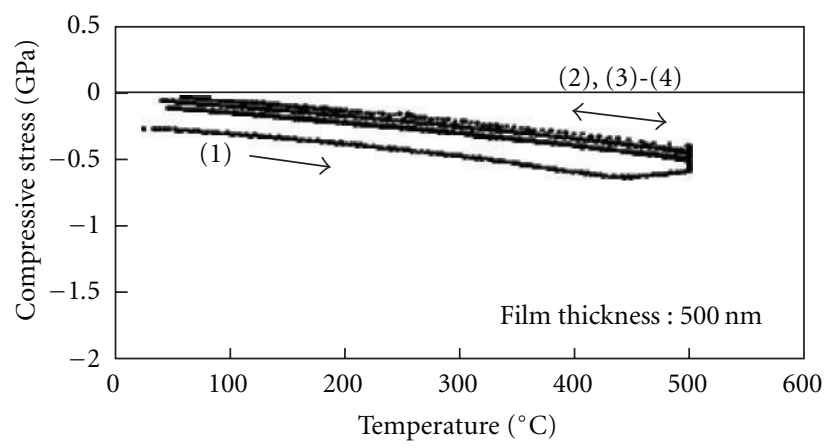

(d)

FIGURE 4: Residual stress behavior during heat-cycle testing (room temp: $-500^{\circ} \mathrm{C}$ ) in an atmosphere of flowing Ar gas for GZO films formed by the RPD system.

indicates that high strain or irregular crystalline structures exist until a far distance from the substrate surface in the film. This is consistent with the result for the dependence of the residual stress on the thickness in the $\mathrm{rf}+\mathrm{dc}$ MS film prior to annealing, as mentioned previously in Figure 2 . In the case of the film formed by the $\mathrm{rf}+\mathrm{dc}$ MS, the irregularity, strain or dispersion of the $c$-axis direction perpendicular to the substrate surface was seen above $500 \mathrm{~nm}$ from the interface.

The behavior of the residual stress due to heat treatment can yield the coefficient of thermal expansion value of a film. For obtaining the coefficient, Young's modulus of the GZO thin-film needs to be determined by some measurement technique. Furthermore, the thermal stress in the film has to be derived from the dependence of the residual stress on the temperature. The residual stresses in the GZO films formed by magnetron sputtering as a function of temperature changed in a complicated manner compared with the residual stress in the GZO film formed by RPD during heating in the first cycle (step (1)), as shown in Figures 4 and 5 . Hence, we focus on the mechanical properties of the GZO thin film formed by the RPD system in this paper. We propose a new technique for the measuring Young's modulus of ultra-thin films and demonstrate its effectiveness for analyzing mechanical characteristics of a film in the present stage.

\subsection{Young's Modulus of GZO Film}

3.2.1. Proposed New Technique for Measuring Young's Moduli of Ultra-Thin Films. Current methods of measuring Young's modulus that are used widely are destructive techniques that damage the samples. In any case, Young's modulus of ultrathin films cannot be measured by these methods. We propose a new nondestructive measurement method for Young's modulus that does not damage the sample and is applicable for ultra-thin films with thickness in the range of approximately $10 \mathrm{~nm}$. Furthermore, we discuss the mechanical characteristics of GZO films obtained using the new method.

Young's modulus $(E)$ has been conventionally determined by techniques measuring the distortion caused in a sample by an external applied force $(F)$ using the following formula:

$$
E=\left(\frac{F}{A}\right) /\left(\frac{\Delta l}{l}\right)
$$

where $A$ is the unit area on the cross-section of the sample perpendicular to the direction of the applied force (load), $\Delta l$ is the expanded length, and $l$ is the original length of the sample. Various traditional techniques using this relationship have been applied for measuring Young's modulus for a long time. The nanoindentation method [12], as shown in Figure 6, has become the major technique for measuring Young's modulus of thin films. However, the method is not applicable to films thinner than ca. $1 \mu \mathrm{m}$. The front edge of a Berkovich tip that is usually employed in depth-sensing indentation systems penetrates the film that easily reaches the vicinity of the under-layer such as a substrate. This causes the penetration depth of the tip-loading weight (force) relationship to deviate from the intrinsic curve that should be observed in the film material. Thus, Young's modulus derived 

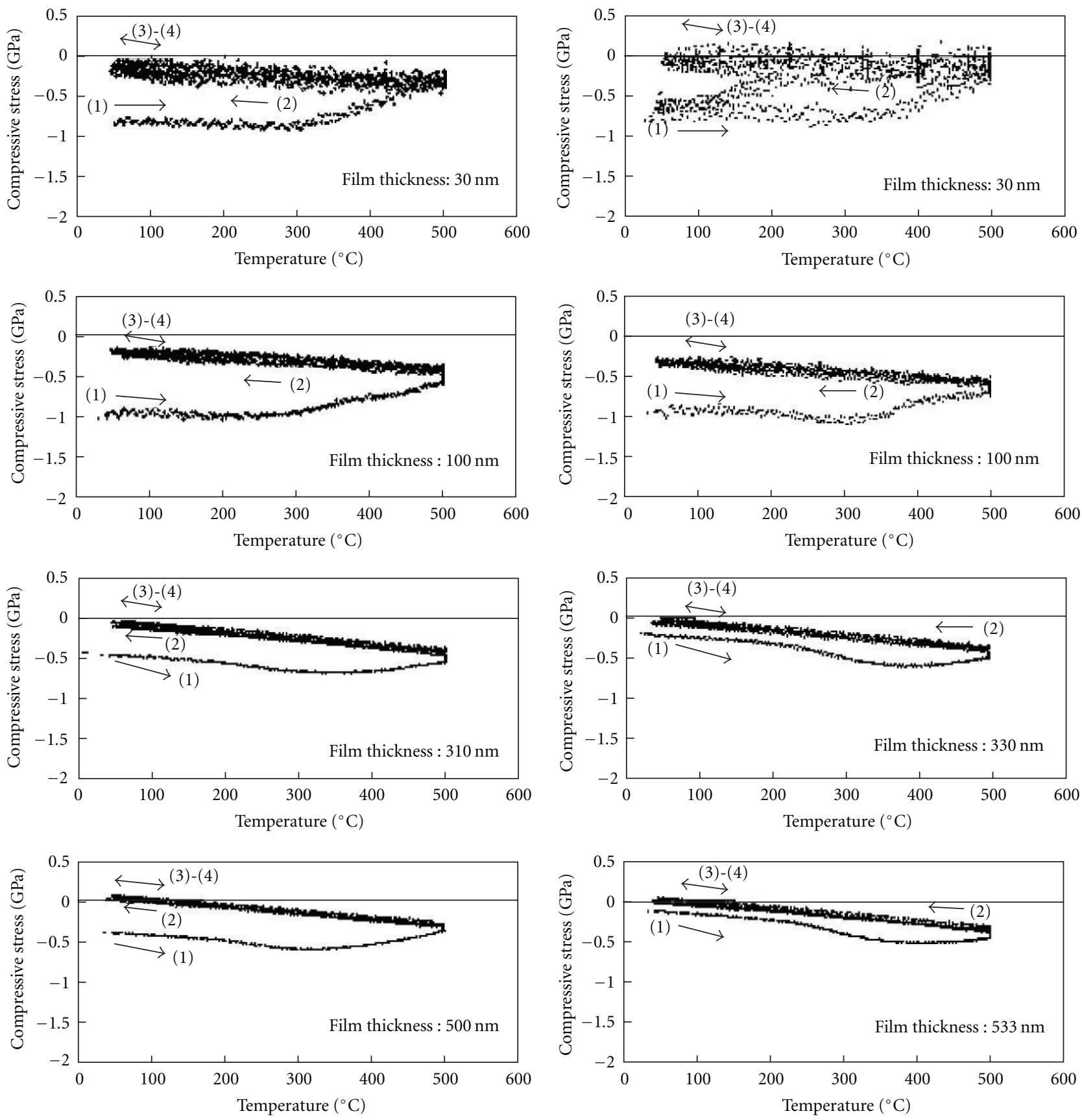

(a)

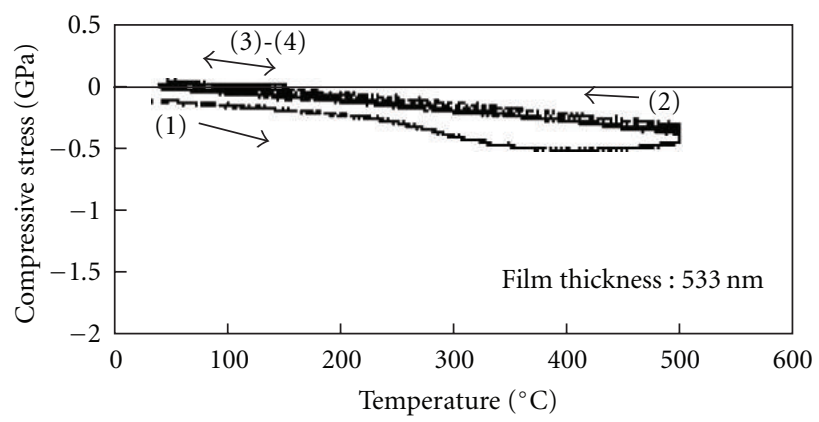

(b)

FIGURE 5: Residual stress behavior during heat-cycle testing (room-temperature: $-500^{\circ} \mathrm{C}$ ) in an atmosphere of flowing Ar gas for GZO films formed by the magnetron sputtering system. The columns (a) and (b) correspond to changes in the residual stress during heat-cycle testing, respectively.

using such a curve deviates from the intrinsic modulus value of the thin film material.

The new technique developed in this work for measuring Young's modulus is applicable for films thinner than $1 \mu \mathrm{m}$. The principle of Young's modulus measurement is shown in Figure 7. $(F / A)$ and $(\Delta l / l)$ in formula (1) correspond to the stress $(\sigma)$ and strain $(\varepsilon)$, respectively. Thus, formula (1) can be rewritten as

$$
E=\frac{\sigma}{\varepsilon}
$$

This is the basic equation of Hooke's law indicating that Young's modulus of a material in a thin film form can be determined directly by measuring both stress and strain within the film.

Generally, the residual stress in a film is measured by the optical lever method, the $\sin ^{2} \psi$ method [13] using X-ray diffraction, or the nanoindentation method described previously. The $\sin ^{2} \psi$ method is not suitable for analyzing the residual stress in a thin film with thickness less than a few micrometers. 


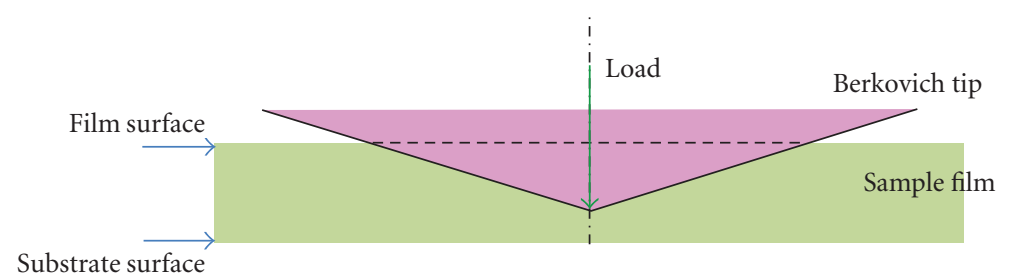

(a) Indent cross-sectional view in a film

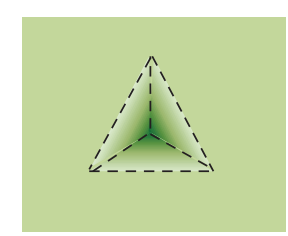

(b) Indent view in the plane of the film

FIGURE 6: Diagram of an indent left by a Berkovich tip in a film.

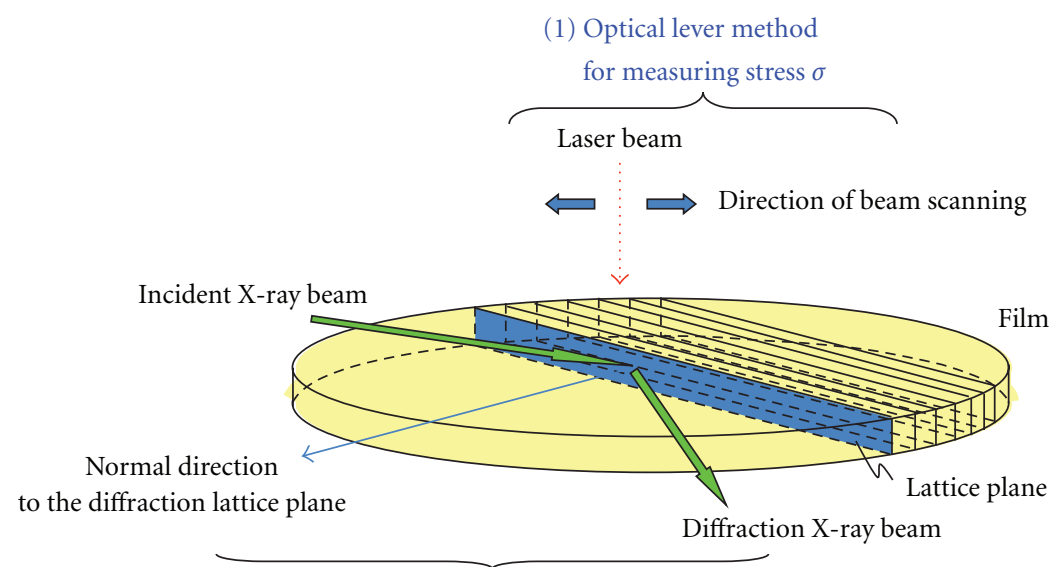

(2) In-plane X-ray diffraction method

for measuring strain $\varepsilon$ in the direction of the film

FIGURE 7: Diagram of a nondestructive measurement technique of Young's modulus and coefficient of linear thermal expansion of a polycrystalline film which uses the optical lever technique for measuring the residual stress in conjunction with the in-plane X-ray diffraction technique for measuring strain.

The stress measured by this technique using the optical lever method is the in-plane component of the residual stress in the film. The strain was measured using grazingincidence wave-dispersive XRD (sometimes referred to as "in-plane X-ray diffraction" [14]). A Cu-K $\alpha$ X-ray (wavelength: $0.154184 \mathrm{~nm}$ ) beam was irradiated with a low angle of incidence on the surface of the sample. This angle was set to 0.35 degrees with respect to the surface, which is close to the total reflection angle for a $\mathrm{ZnO}$ film. The strains and grain sizes along the in-plane direction in each film were obtained by the Williamson-Hall equation (plot) [15], shown hereinafter, using the angles and the full widths at half maxima (FWHMs) of the diffraction X-ray peaks with crystalline indexes of the material of the film:

$$
\frac{\sigma \cos \theta}{\lambda}=2 \varepsilon\left(\frac{\sin \theta}{\lambda}\right)+\left(\frac{1}{\delta}\right),
$$

where $\sigma$ is the full width at half maximum (FWHM) of the diffraction X-ray peak, $\theta$ is the angle of X-ray diffraction peak, $\varepsilon$ is the strain, and $\delta$ is the grain size.

Therefore, when $\sigma \cos \theta / \lambda$ (vertical axis: $y$-axis) as a function of $\sin \theta / \lambda$ (horizontal axis: $x$-axis) is plotted, the strain is obtained from the slope of the $y$ - $x$ curve and the grain size is determined from the $y$-intercept.
Our proposed technique for measuring Young's modulus combines the previous two techniques. It has the following special features: (1) It is nondestructive (the sample is not damaged), (2) it is applicable for ultra-thin films with thickness as small as ca. $10 \mathrm{~nm}$, (3) it allows for the value of each layer in a multilayer composite sample to be obtained separately, and (4) it can determine Young's modulus in the direction of a crystalline index. (This is fundamentally possible in the optical lever method; however, it can typically only measure the residual stress in the direction of the plane of the film. It then becomes necessary to introduce a method of enabling vector analysis on the stress.)

\subsubsection{Strain and Grain size in GZO Polycrystalline Ultra-Thin} Films. The diffraction patterns from GZO films prepared by the RPD system obtained using out-of-plane and in-plane techniques for XRD measurements are shown in Figure 8. Only the (002) and (004) diffraction peaks of $\mathrm{ZnO}$ with a Wurtzite crystalline structure in the diffraction data were obtained by the out-of plane XRD technique, as shown in Figure 8(a). According to Figure 8(b), the peaks obtained by the in-plane XRD technique were diffracted from the $(\mathrm{xx} 0)$ crystalline planes. These results indicate that the GZO polycrystalline films consist of grains with the $c$-axes of the 


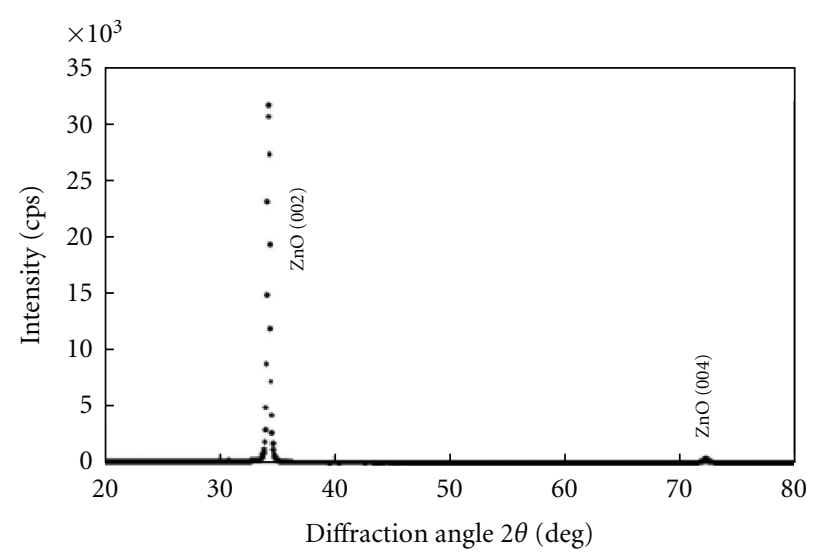

(a) Out-of-plane

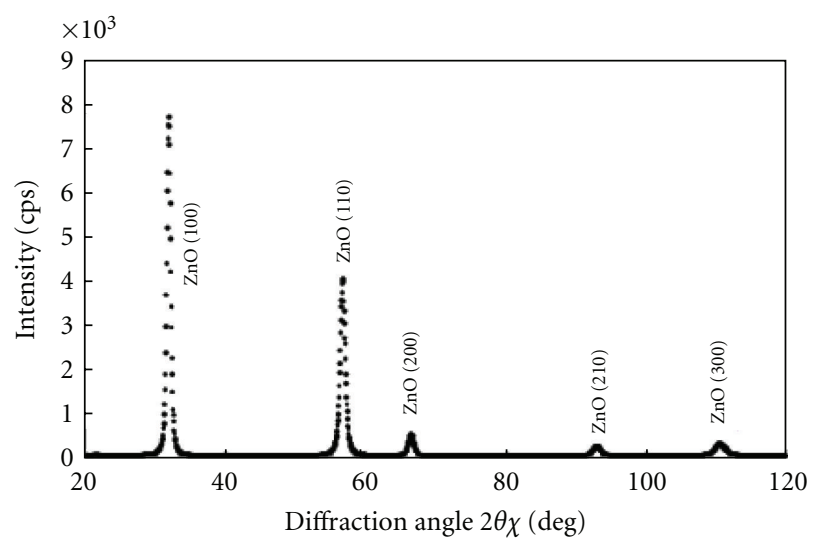

(b) In-plane

Figure 8: Typical XRD profile of a GZO film with $150 \mathrm{~nm}$ thickness prepared at $180 \circ \mathrm{C}$ using the RPD system. Profiles (a) and (b) were obtained using out-of-plane and in-plane techniques for XRD measurements, respectively.

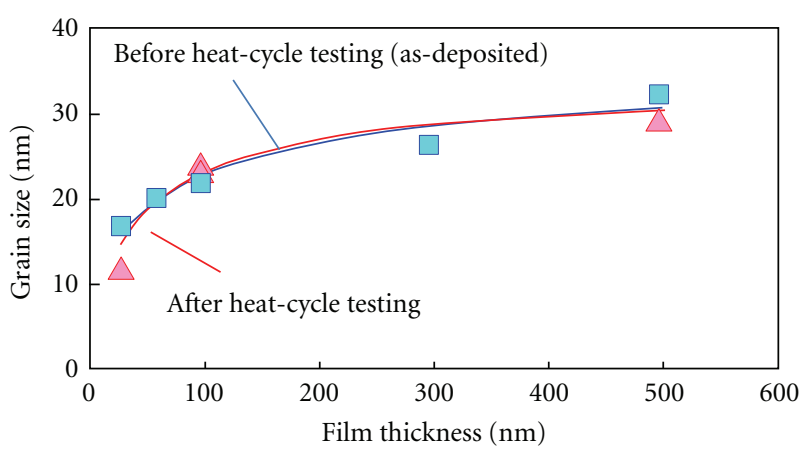

(a) Grain size

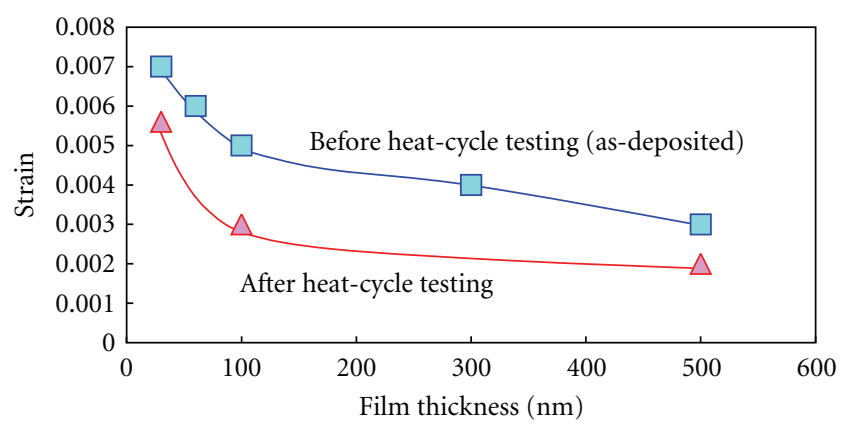

(b) Strain

FIgURE 9: Average grain size and strain in the in-plane direction ( $a$-axis of Wurtzite cell) as a function of film thickness.

Wurtzite crystalline cells oriented strictly along the normal direction to the surface of the film.

The strains and grain sizes in polycrystalline GZO films were determined using (3) and the (x00) diffraction peaks in the in-plane XRD patterns, respectively. The obtained strain is the component in the $a$-axis direction of the Wurtzite crystalline cell of $\mathrm{ZnO}$, that is, in the in-plain direction of the GZO film. Figure 9 shows the dependence of strain and grain size in a GZO film on the film thickness before and after the heat-cycle testing in the range of room temperature to $500^{\circ} \mathrm{C}$. The grain size increased monotonically with the film thickness. On the other hand, the strain decreased with increasing film thickness. These dependencies on the film thickness had good correspondence to cross-sectional TEM micrographs shown previously in Figure 1. That is, region (1) close to the interface of the substrate had smaller grains and higher strains caused because the direction of stacking of alternative $\mathrm{Zn}$ and $\mathrm{O}$ atomic layers (the direction coincided with that of the $c$-axis of the Wurtzite crystalline cell) was not corresponding between each crystalline grain. To compare with it, the stacking directions were precisely oriented among grains in region (2) at a far distance from the surface of the substrate as shown in Figure 1. In particular, the dependence of strain on film thickness was consistent with the dependence of the regular stacking of atomic layers on the distance from the interface. The strain characteristics as a function of the GZO thickness in region (1) in Figure 1 had a slope steeper than that in region (2).

We next discuss changes in the grain size and strain in a GZO film caused by the heat-cycle testing between room temperature and $500^{\circ} \mathrm{C}$. The heat treatment had little or no effect on grain growth in the in-plane direction (a-axis direction). On the other hand, the strain in the GZO film decreased remarkably by the heat-cycle testing. The reduction rate of strain in the film with ca. $100 \mathrm{~nm}$ thickness caused by the heat-cycle testing reached about $50 \%$. On the other hand, strain in a very thin film with ca. $30 \mathrm{~nm}$ thickness was reduced only about $20 \%$ by the heat treatment. It is estimated that the heat-cycle testing condition was inadequate for releasing high strain caused by highly crystalline irregularities in region (1). It is considered that a large number of crystalline defects were included in the vicinity of the interface with the substrate, such as in region (1) in Figure 1(a).

3.2.3. Young's Modulus of GZO Thin Film. Young's modulus of a GZO thin film was derived by substituting stress and strain into (2) as mentioned in the previous section. Young's modulus of the as-deposited GZO film depended on the film thickness as shown in Figure 10(a). Young's modulus of 
an ultra-thin film, such as with $30 \mathrm{~nm}$ thickness, was about $205 \mathrm{GPa}$. The film with $60 \mathrm{~nm}$ thickness had a maximum modulus of $300 \mathrm{GPa}$. This value decreased with increasing thickness of the film and reached a value of about $117 \mathrm{GPa}$ in the comparatively thick GZO film with $500 \mathrm{~nm}$ thickness. Generally, Young's modulus shows a correlation with the hardness of a material. The hardness increases with the number of defects in a material. The defects pin atomic layer slip relative to other layers in a crystal due to an external force. Then, Young's modulus in an ultra-thin film or in the interfacial neighborhood with a large number of defects becomes larger than that of a thick film or at a far distance from the surface of a substrate. This dependence shown in Figure $10(a)$ is consistent with that of the distribution of crystalline irregularities or the dispersion of the stacking direction of atomic layers within each grain shown in Figures 1(a) and 1(b). The dependence of Young's modulus of a GZO thin film on the film thickness after heat-cycle testing between room temperature and $500^{\circ} \mathrm{C}$ is also shown in Figure 10(b). Young's modulus of the comparatively thicker film with $500 \mathrm{~nm}$ thickness was reduced remarkably by about one digit and half from the value before heat-cycle testing. The reason why Young's modulus of the thicker film with relatively fewer defects decreased drastically is not yet clear. In an area away from the interface with the substrate, as shown in Figure 1(c), the $\mathrm{Zn}$ and $\mathrm{O}$ atomic layers at the polycrystalline grain boundary were smoothly connected. The proportion of the volume of the region where a large number of defects existed in the vicinity of the surface of the substrate in the total volume of the film decreased with the increasing thickness of the film. Then, the value obtained for Young's modulus was affected by the ratio of region $(2) /($ region $(1)+$ region $(2))$ as shown in Figure 1 . In the case of the $500 \mathrm{~nm}$-thick GZO film, Young's modulus measured by this new technique was almost equal to that of region (2) with small amount of defects, since the volume of the region became one order of magnitude larger than that of region (1) with a large number of defects. At this stage, we can only speculate on the reason for why Young's modulus of the $500 \mathrm{~nm}$ thick was reduced significantly by heat-cycle testing. Very small numbers of defects existed at grain boundaries in region (2), which was one of the main factors contributing to the modulus before heat treatment. The positions that the $\mathrm{Zn}$ and $\mathrm{O}$ atomic layers were connected continuously smoothly at the grain boundaries as shown in region (2) in Figures $1(\mathrm{a})$ and 1 (c) and the positions that they were not connected smoothly in region (1) existed in a film. Then, the crystalline defects occurred at the later positions in the grain boundaries. This small number of defects in region (2) could disappear easily from the grain boundaries by heat treatment. As a result, Young's modulus of thick films, such as with $500 \mathrm{~nm}$ thickness, was reduced remarkably by heat treatment. The value was almost comparable with that of a material with low hardness, such as talc or calcium sulfate.

\subsection{Coefficient of Linear Thermal Expansion of GZO Films.} The thermal stress $\left(\sigma_{T}\right)$ in a film is derived by the following equation using the temperature $(T)$ dependence of residual stress in the film:

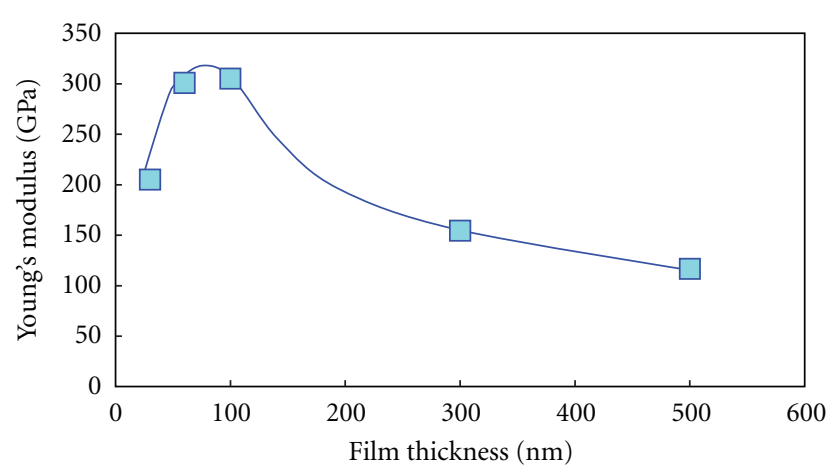

(a)

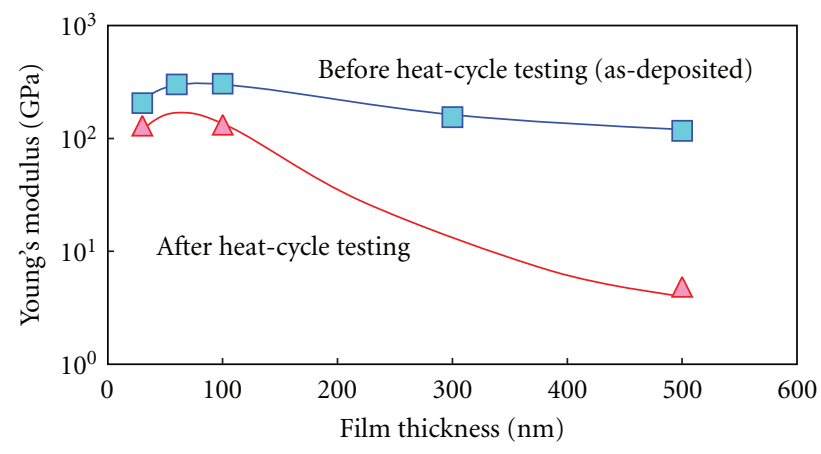

(b)

Figure 10: Young's modulus in the in-plane direction ( $a$-axis of Wurtzite cell) of the GZO film formed by the RPD system as a function of film thickness. (a) Young's modulus before heat-cycle testing (as-deposited). (b) Reduction in Young's modulus value of the thin film caused by the heat-cycle testing. The same data in (a) is redrawn in (b).

$$
\sigma_{T}=\frac{\Delta \sigma}{\Delta T} .
$$

Using the thermal stress $\left(\sigma_{T}\right)$ and Young's modulus of the film, the coefficient of linear thermal expansion $\left(\alpha_{f}\right)$ of the film can be derived by the following:

$$
\alpha_{f}=\frac{\sigma_{T}\left(1-v_{f}\right)}{E_{f}}+\alpha_{s} .
$$

Here, $v_{f}, E_{f}$, and $\alpha_{s}$ represent the Poisson's ratio of the film, Young's Modulus of the film, and the coefficient of linear thermal expansion of the substrate, respectively.

The thermal stress and the coefficient of linear thermal expansion of the GZO films formed on the thermally oxidized Si wafer of a single crystal with a (100) crystalline surface were obtained using the stress-temperature characteristics of the GZO film shown in Figure 4 and using (4) or (5). These material properties of the GZO films were derived from the stress-temperature characteristics in the range between room temperature and $250^{\circ} \mathrm{C}$ taking into account the following factors: (1) Young's moduli of the GZO films derived using the measured residual stresses and strains in the films mentioned in the previous section were values at room temperature, and (2) the highest processing 


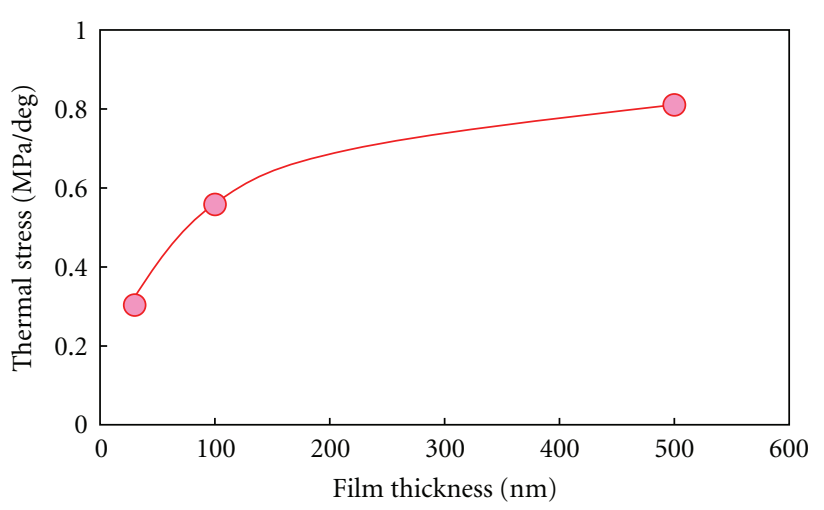

(a)

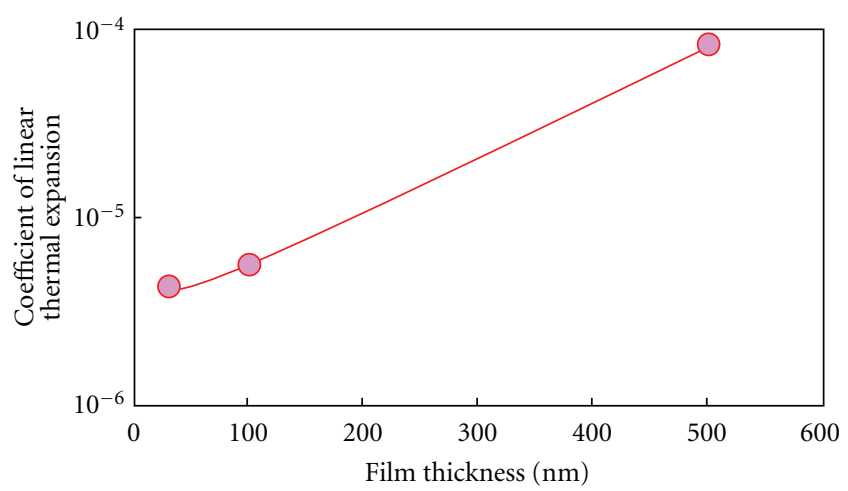

(b)

FIgURE 11: Thermal stress and coefficient of linear thermal expansion of GZO films formed by the RPD system as a function of film thickness. (a) Dependence of thermal stress on film thickness, and (b) dependence of the coefficient of linear thermal expansion on film thickness.

temperature for manufacturing LCDs is generally limited to about $250^{\circ} \mathrm{C}$ since parts such as RGB (Red-Green-Blue) color filters that form LCDs are made of organic materials. As shown in Figure 4, when the samples were heated up in the first cycle step (step (1)), the behavior of the residual stress as a function of the temperature was complicated. Then, the dependence of the residual stress on the temperature on the second heating stage (step (3)) was used for obtaining the thermal stress.

The value of $2.67 \times 10^{-6}{ }^{\circ} \mathrm{C}^{-1}$ at around $100^{\circ} \mathrm{C}$ for the coefficient of linear thermal expansion of $\mathrm{Si}$ as function of temperature, reported by Okada and Tokumura [16], was used for $\alpha_{s}$ in (5). The thermal stress and the coefficient of linear thermal expansion of a GZO film were then determined as shown in Figure 11. The Poisson ratio of a GZO film was tentatively assigned as 0.3 , which is the average of the Poisson ratios of $\mathrm{ZnO}$ picked as obtained from various reports [17-23], because our technique had not yet reached an applicable level to measure it in this stage. In the case of a Poisson ratio of 0.1 lower or higher than 0.3 (Poisson ratio: 0.2 or 0.4 ), the coefficient of linear thermal expansion increases or decreases only about $7 \%$.

The coefficients of linear thermal expansion of the GZO films with 30-100 nm thickness coincided with our expectation. On the other hand, the coefficient of linear thermal expansion of the $500 \mathrm{~nm}$ thick film deviated far from our expectation. It is not clear why an abnormal coefficient of linear thermal expansion of the film with $500 \mathrm{~nm}$ thickness was obtained. We believe that this likely originated in the excellent continuity of atomic layers at polycrystalline boundaries, similar to a singly crystal as shown in Figure $1(\mathrm{c})$.

\section{Summary}

We developed a new technique for analyzing the mechanical properties of ultra-thin polycrystalline films. This method for measuring Young's modulus of an ultra-thin film used stress measurement by the optical lever technique together with X-ray diffraction with an X-ray beam incident from an approximately total reflection angle (in-plane XRD technique). This measurement technique is nondestructive and does not cause any damage to a film. Furthermore, the technique can be applied for measuring Young's modulus of ultra-thin films of various polycrystalline materials with 10$30 \mathrm{~nm}$ thickness.

In the dependence of the residual stress in the GZO thin films on film thickness, the maximum stress appeared in the film with $50-60 \mathrm{~nm}$ thickness. This thickness coincided exactly with the distance from the surface of the substrate, that changes from the region with irregular stacking directions of alternate $\mathrm{Zn}$ and $\mathrm{O}$ atomic layers among columnar grains to the region consisting of grains with strictly aligned stacking direction. Thus, the residual stress represents information from nanoscale regions in crystalline films.

Young's moduli of the GZO films showed a similar dependence on the film thickness. The maximum modulus reached a value of about $300 \mathrm{GPa}$. The modulus decreased with increasing thickness. The GZO film with $500 \mathrm{~nm}$ thickness had a Young's modulus of $117 \mathrm{GPa}$. This value of the modulus is reasonable compared to values of $61-125 \mathrm{GPa}$ reported for $\mathrm{ZnO}$ films with $2-3 \mu \mathrm{m}$ thickness measured using the nanoindentation technique [24]. The coefficient of linear thermal expansion of GZO films with $30-100 \mathrm{~nm}$ thickness was in the range of $4.3 \times 10^{-6}-5.6 \times 10^{-6}{ }^{\circ} \mathrm{C}^{-1}$. On the other hand, the film with $500 \mathrm{~nm}$ thickness had a remarkable high coefficient of $8.3 \times 10^{-5}{ }^{\circ} \mathrm{C}^{-1}$. We estimated that this high value was related to the excellent continuity of $\mathrm{Zn}$ and $\mathrm{O}$ atomic layers in the in-plane direction (the direction of the $a$-axis of the Wurtzite cell of $\mathrm{ZnO}$ ) between grain boundaries similar to a single crystal. This excellent continuity also had a favorable effect on the low Young's modulus and low residual stress.

The results indicate that the new measurement technique for Young's modulus of ultra-thin films is very useful. The mechanical properties of the GZO films formed by conventional magnetron sputtering are not discussed in this paper. In a future paper, the characteristics of the films will be compared with those of the GZO films prepared by the RPD system. Furthermore, the technique will be applied for developing $\mathrm{ZnO}$ transparent conductive films that can resist external mechanical pressures during the manufacture of LCDs and testing long-term reliability of 
displays under external conditions of heating at $50-60^{\circ} \mathrm{C}$ and a high humidity of $90-95 \%$.

\section{Acknowledgments}

The authors would like to thank Professor Dr. Y. Yasuda of Center for Interdisciplinary Research, Tohoku University, Dr. T. Yamada of Guraduate school of Engineering, Osaka University and Mr. M. Hakata of a former board member of CASIO Computer Co., Ltd., for their encouragement, contributions and suggestions during the author's research on the mechanical properties of ultra-thin films. The authors also thank Dr. K. Takeda, Mr. T. Mine, and Mr. N. Sakuma of the Central Research Laboratory of Hitachi Co. Ltd. for providing access to their analytical facilities, and Dr. K. Inaba of the X-ray Research Laboratory of Rigaku Corporation for valuable advice throughout the course of this work. This work was made possible by a grant for Collaboration of Regional Entities for the Advancement of Technological Excellence from the Japan Science and Technology Agency, and it was supported in part by a grant for the Development of Indium Substitute Materials for a Transparent Conducting Electrode in Rare Metal Substitute Materials Development Project from NEDO (New Energy and Industrial Technology Development Organization).

\section{References}

[1] D. Kempthorne and M. D. Myers, Mineral Commodity Summaries 2007, United States Government Printing Office, Washington, DC, USA, 2007.

[2] S. Homma, A. Miyamoto, S. Sakamoto, K. Kishi, N. Motoi, and K. Yoshimura, "Pulmonary fibrosis in an individual occupationally exposed to inhaled indium-tin oxide," The European Respiratory Journal, vol. 25, no. 1, pp. 200-204, 2005.

[3] T. Yamamoto, T. Yamada, A. Miyake, H. Makino, and N. Yamamoto, "Ga-doped zinc oxide: an attractive potential substitute for ITO, large-area coating, and control of electrical and optical properties on glass and polymer substrates," Journal of the Society for Information Display, vol. 16, no. 7, pp. 713-719, 2008.

[4] R. S. Wagner, A. K. Sinha, T. T. Sheng, H. J. Levinstein, and F. B. Alexander, "Tungsten metallization for LSI applications," Journal of Vacuum Science and Technology, vol. 11, pp. 582590, 1974.

[5] J. A. Thornton and D. W. Hoffman, "Internal stresses in titanium, nickel, molybdenum, and tantalum films deposited by cylindrical magnetron sputtering," Journal of Vacuum Science and Technology, vol. 14, pp. 164-168, 1977.

[6] D. W. Hoffman and J. A. Thornton, "The compressive stress transition in $\mathrm{Al}, \mathrm{V}, \mathrm{Zr}, \mathrm{Nb}$ and $\mathrm{W}$ metal films sputtered at low working pressures," Thin Solid Films, vol. 45, no. 2, pp. 387396, 1977.

[7] H. Windischmann, "Intrinsic stress in sputter-deposited thin films," Critical Reviews in Solid State and Materials Sciences, vol. 17, no. 6, pp. 547-596, 1992.

[8] N. Yamamoto, H. Kume, S. Iwata et al., "Fabrication of highly reliable tungsten gate MOS VLSI's," Journal of the Electrochemical Society, vol. 133, no. 2, pp. 401-407, 1986.

[9] R. E. Cuthrell, D. M. Mattox, C. R. Peeples, P. L. Dreike, and K. P. Lamppa, "Residual stress anisotropy, stress control, and resistivity in post cathode magnetron sputter deposited molybdenum films," Journal of Vacuum Science and Technology A, vol. 6, no. 5, pp. 2914-2920, 1988.

[10] N. Yamamoto, T. Yamada, A. Miyake, H. Makino, S. Kishimoto, and T. Yamamoto, "Relationship between residual stress and crystallographic structure in Ga-doped ZnO film," Journal of the Electrochemical Society, vol. 155, no. 9, pp. J221-J225, 2008.

[11] N. Yamamoto, H. Makino, T. Yamada et al., "Heat resistance of Ga-doped $\mathrm{ZnO}$ thin films for application as transparent electrodes in liquid crystal displays," Journal of the Electrochemical Society, vol. 157, no. 2, pp. J13-J20, 2010.

[12] E. B. Tadmor, R. Miller, and R. Phillips, "Nanoindentation and incipient plasticity," Journal of Materials Research, vol. 14, no. 6, pp. 2233-2250, 1999.

[13] P. S. Prevey, "X-ray diffraction residual stress techniques," in Metals Handbook, vol. 10, pp. 380-392, American Society for Metals, Metals Park, Ohio, USA, 1986.

[14] B. K. Tanner, T. P. A. Hase, T. A. Lafford, and M. S. Goorsky, "Grazing incidence in-plane X-ray diffraction, international centre for diffraction data," Advances in X-Ray Analysis, vol. 47, pp. 309-314, 2004.

[15] G. K. Williamson and W. H. Hall, "X-ray line broadening from filed aluminium and wolfram," Acta Metallurgica, vol. 1, no. 1, pp. 23-31, 1953.

[16] Y. Okada and Y. Tokumura, "Precise determination of lattice parameter and thermal expansion coefficient of silicon $300 \mathrm{~K}-$ 1500 K," Journal of Applied Physics, vol. 56, no. 2, pp. 314-320, 1984.

[17] S. Imai, M. Tokuyama, S. Hirose, G. J. Burger, T. S. J. Lammerink, and J. H. J. Fluitman, "A thin-film piezoelectric impact sensor array fabricated on a Si slider for measuring head-disk interaction," IEEE Transactions on Magnetics, vol. 31, no. 6, pp. 3009-3011, 1995.

[18] H. K. Yoon and Y. S. Yu, "Hardness and elastic modulus of $\mathrm{ZnO}$ thin films fabricated by PLD method," Key Engineering Materials, vol. 353-358, no. 4, pp. 2966-2969, 2007.

[19] I. Özen, M. A. Gülgün, and M. Özcan, "Self-induced crystallinity in RF magnetron sputtered $\mathrm{ZnO}$ thin films," Key Engineering Materials, vol. 264-268, pp. 1225-1228, 2004.

[20] H. C. Jung, H. K. Yoon, and Y. S. Yu, "Mechanical properties and process of $\mathrm{ZnO}$ deposited various substrates," Key Engineering Materials, vol. 297-300, pp. 533-538, 2005.

[21] G. Cao and X. Chen, "Size dependence and orientation dependence of elastic properties of $\mathrm{ZnO}$ nanofilms," International Journal of Solids and Structures, vol. 45, no. 6, pp. 1730-1753, 2008.

[22] G. Stan, C. V. Ciobanu, P. M. Parthangal, and R. F. Cook, "Diameter-dependent radial and tangential elastic moduli of ZnO nanowires," Nano Letters, vol. 7, no. 12, pp. 3691-3697, 2007.

[23] O. Kappertz, R. Drese, and M. Wuttig, "Correlation between structure, stress and deposition parameters in direct current sputtered zinc oxide films," Journal of Vacuum Science and Technology A, vol. 20, no. 6, pp. 2084-2095, 2002.

[24] T.-H. Fang, W.-J. Chang, and C.-M. Lin, "Nanoindentation characterization of $\mathrm{ZnO}$ thin films," Materials Science and Engineering A, vol. 452-453, pp. 715-720, 2007. 

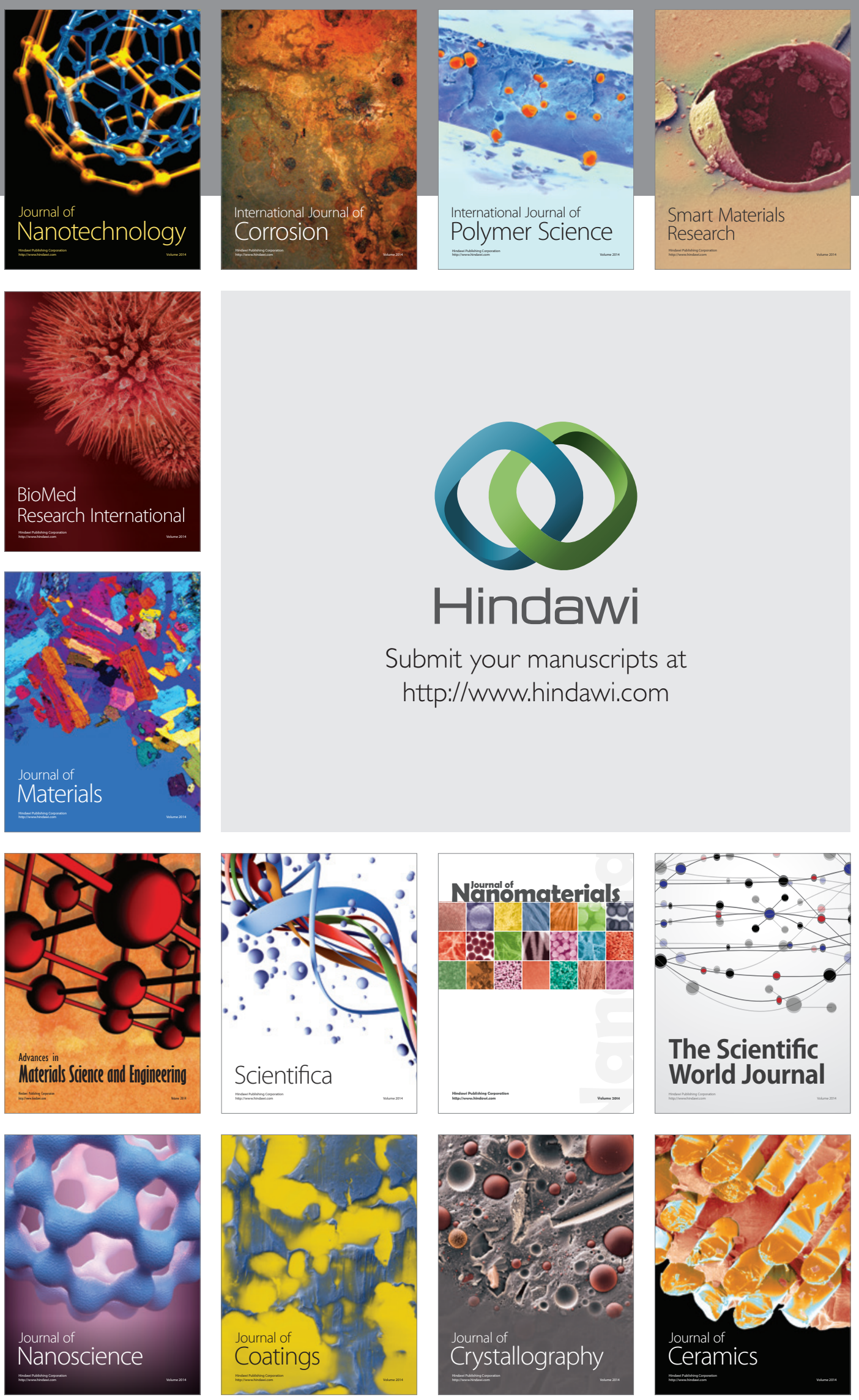

The Scientific World Journal

Submit your manuscripts at

http://www.hindawi.com

\section{World Journal}

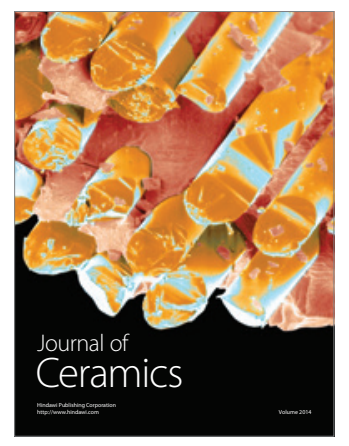

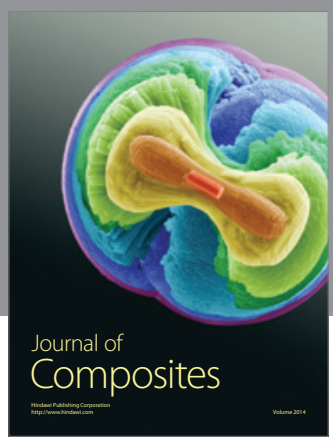
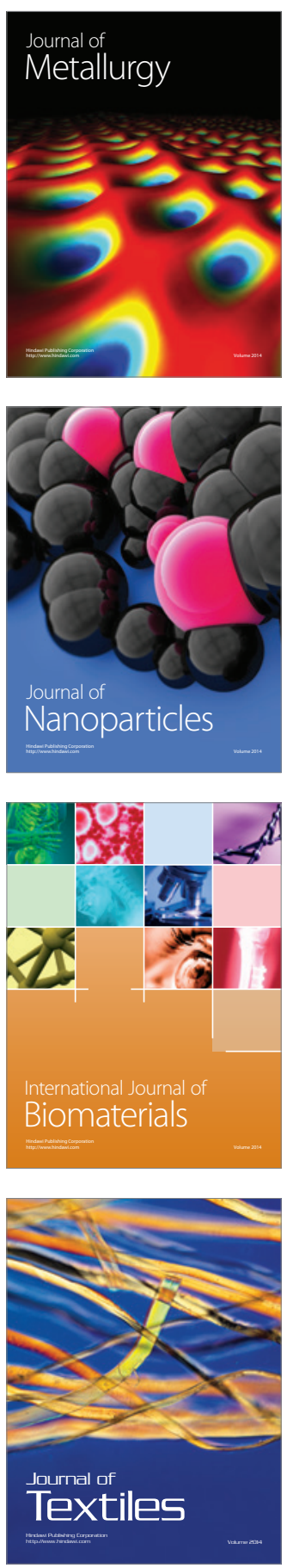